\title{
Estudio confirmatorio acerca de la frecuencia y percepción de la violencia: El VIDOFyP como instrumento de evaluación y algunas reflexiones psicosociales
}

\section{Confirmatory study about the frecuency and perception of the violence: The VIDOFyP like an evaluation instrument and some psychosocial reflections}

\author{
P. TRUJANO* \\ C. NAVA* \\ E. TEJEDA* \\ S. GUTIÉRREZ*
}

\begin{abstract}
RESUMEN
Este trabajo tuvo como objetivo confirmar la utilidad, confiabilidad y validez del instrumento llamado VIDOFyP (Violencia Doméstica: Frecuencia y Percepción) (Mendoza y Trujano, 1998; en Trujano y Mendoza, 2003), para identificar qué actitudes y comportamientos perciben como violentos en una situación de pareja mujeres de diferentes edades y niveles educativos, y saber con qué frecuencia y modalidades se presentan; a partir de esta información, conocer la posible correlación entre la percepción de conductas violentas y su ocurrencia, replicando dos estudios anteriores que conforman una investigación más amplia.

La muestra se integró con 200 mujeres divididas en dos Estudios. En el primero se incorporó a 50 mujeres adolescentes y a 50 jóvenes; en el segundo a 50 mujeres con nivel educativo profesional (licenciatura) y a 50 con un nivel básico (primaria). La información se obtuvo aplicando el VIDOFyP, y permitió observar la presencia de comportamientos violentos en ambos estudios. En general, las mujeres jóvenes y las de nivel profesional mostra-
\end{abstract}

\footnotetext{
* Carrera de Psicología, Facultad de Estudios Superiores Iztacala, Universidad Nacional Autónoma de México.
} 
ron una mayor percepción de la violencia. La modalidad de violencia mejor percibida en ambos estudios fue la psicológica; una mayor experiencia y un grado más alto de escolaridad parecen facilitar (aunque no garantizar) una mayor percepción y una menor frecuencia de comportamientos violentos. El instrumento mostró su utilidad al observarse niveles satisfactorios de confiabilidad y validez.

\title{
PALABRAS CLAVE
}

Violencia Doméstica, Género, Victimización.

\begin{abstract}
The current job had as objective to confirm the utility, reliability and validity of the instrument named VIDOFyP (Violencia Doméstica: Frecuencia y Percepción) (Mendoza y Trujano, 1998; en Trujano y Mendoza, 2003), to identify what attitudes and behaviors are perceived like violent in a couple of women of different ages and educative level situations, and also knowing with what frequency are presented, and from this information, to know the possible relationship between the violent behavior perception and it's happening, replying two previous studies part of a bigger investigation.

The sample was formed by 200 women divided in two studies. In the first one, there were incorporated fifty teenagers women and fifty young women; and in the second one, fifty women with a high professional level (degree) and fifty with a basic level (primary school). The information was found by using the VIDOFyP, and it allowed to see the presence of violent behaviors in both studies. In general, the young women and the women with a professional level showed a bigger perception of violence. The violence modality better perceived in both studies was the psychological one. A high experience and a high professional level apparently provide (although no guarantee) the bigger perception and the fewer frequency of violent behavior. The instrument showed its utility by been observed satisfactory level of reliability and validity.
\end{abstract}

\section{KEY WORDS}

Domestic Violence, Gender, Victimization. 


\section{ANTECEDENTES}

\section{La violencia como problema social}

La violencia constituye uno de los problemas cada vez más frecuentes en nuestros días, e irrumpe un espacio tradicionalmente concebido como de mayor seguridad y bienestar, el hogar, al cual se le conoce como Violencia Doméstica. $\mathrm{Al}$ respecto, Giddens (2000c) señala que el análisis de género ha mostrado que es una forma de dominación que incluye el control y el ejercicio del poder incluso en los ámbitos de la intimidad, tales como el familiar, el conyugal y el sexual. Y aunque tradicionalmente el generador de la violencia suele ser el varón, y las víctimas las mujeres, niños y ancianos, el abuso puede provenir de cualquiera de los miembros; además, se presenta en todos los estratos sociales, no siendo determinante, la etnia, el credo, ni el régimen político.

Desde la perspectiva de género se admite el carácter relacional de las interacciones genéricas, y por ende, la consideración de que, aunque ciertamente las mujeres siguen siendo las más victimizadas, actualmente (quizás por el periodo de transición que estamos viviendo), cada vez más los combates son mutuos, y también, cada vez más hombres son victimas de la violencia de sus mujeres (Trujano, Martínez y Benítez, 2002), lo que puede observarse en el incremento de sus denuncias, aunque todavía se hable muy poco de esta cara del fenómeno. Ello nos lleva a estudiar la violencia como un hecho deplorable (sobre todo por sus efectos en el entorno familiar) que requiere de programas de prevención e intervención urgentes que permitan fortalecer el equilibrio intrafamiliar del poder, propio de la democracia familiar (Giddens, 2000aํ), en el que se sostiene a su vez, la consolidación de la democracia social y política (Trujano, M.; 2003).
Dado que las cifras de violencia en contra de las mujeres siguen siendo las más dramáticas, por ahora nos centraremos en ellas.

\section{Violencia Doméstica}

Muchos estudios han dirigido sus investigaciones a la búsqueda de perfiles del generador de violencia y de la víctima. Nuestra postura es que esta visión ya resulta dificilmente sostenible, pues, aún cuando no podemos descartar la existencia de rasgos de personalidad antisocial en algunas personas, o la presencia de psicopatías en otras, en general, cada vez más estudios señalan la complejidad de la violencia, por lo que la consideramos un fenómeno multiderminado (Trujano, 1994; Corsi, Dohmen, Sotés y Bonino, 1995; Neiding y Friedman, 1988), en el que entran en juego variables que incluyen desde lo psicológico, lo biológico, lo socio-cultural, lo educativo, etcétera. Para autores como Levande, Koch y Koch (1983), la violencia dentro de la pareja suele estar relacionada con la interacción de diferentes factores como la actitud ante la violencia, las circunstancias socioeconómicas y la conducta de los miembros de la díada, así como el sexo y las ideas que sostienen sobre sí mismos y sobre su rol de género. Por otro lado, para Dibble y Strauss (citados en Levande, et.al, 1983) las tasas de violencia doméstica suelen estar relacionadas con aspectos como las creencias previas acerca del uso de la violencia (p.ej., pensar que golpear es normal) y circunstancias como los ingresos, el estatus en el empleo y el sexo.

\section{Relevancia}

Las últimas dos décadas se han caracterizado por un interés creciente por la violencia doméstica, debido quizás 
a su alta incidencia y al daño que puede ocasionar en sus victimas. Sin embargo, las cifras no son contundentes, pues se sigue calculando que solamente un 10\% a $30 \%$ de las víctimas denuncian, y muchas de ellas ni siquiera acuden a centros o asociaciones de ayuda. Por otro lado, un común denominador es que, a pesar de lo grave que puede ser, muchas victimas permanecen con esa pareja durante periodos prolongados de tiempo, más de 10 años por término medio. Incluso, en muchos casos, y tras la intervención terapéutica, se regresa a la relación anterior. Además, el maltrato suele iniciar desde el comienzo de la relación (antes de formalizarla) e ir en aumento tanto en frecuencia como en intensidad. Desafortunadamente, cuanto más tiempo se permanezca dentro de una relación abusiva, la probabilidad de que las consecuencias psicológicas se cronifiquen es mayor, y en consecuencia, el pronóstico de recuperación es más desfavorable (Zubizarreta, Sarasua, Echeburúa, De Corral, Sauca y Emparanza, 1994). Al respecto, en México, datos revelados por el CAVI (Centro de Atención a la Violencia Intrafamiliar) de la Procuraduría General de Justicia del DF apuntan a que en el $72 \%$ de los casos atendidos en los últimos años, se pudo detectar que la violencia se inició desde el noviazgo (Calderón, 1994; Trujano y Mata, 2002). Un problema adicional es que las cifras muestran una lenta y paulatina reversión entre sus protagonistas, pues en 1997 se calculaba que el 1\% de las mujeres maltrataba a sus compañeros; para 1998 la cifra aumentó a un 2\%, y para el 2005 se registran de un 3\% a un $10 \%$ de varones maltratados que denuncian en México, si bien en algunos países europeos hay quien asegura un $20 \%$ y en Estados Unidos hasta un 40\%.También se empieza a observar una mayor tendencia a los combates mutuos (un 23\% aproximadamente), aunque la mayor parte de las denuncias (más 0 menos un $75 \%$ ) siguen siendo de mujeres victimizadas.

\section{Planteamiento}

Lo anterior nos lleva a la necesidad de derivar medidas eficaces, y el punto de partida puede ser tener una perspectiva más clara y abarcativa, pues actualmente la mayoría de las investigaciones se han centrado en la violencia física o en la sexual, quizás por ser las más evidentes y según algunos autores, las más dañinas. Sin embargo, es momento de contemplar otras modalidades que por integrarse de una manera menos drástica a la cotidianeidad, se vuelven más permisibles socialmente, y más fácilmente tolerables. En este sentido, la categorización propuesta por CORIAC (Colectivo de Lucha por Relaciones Igualitarias) en 1998, que incluye la violencia física, sexual, psicológica, financiera, objetal y social, parece responder con mayor justicia a las vivencias de maltrato de muchas personas, y puede permitir un mejor entendimiento de sus posibles manifestaciones y una actitud general de mayor reflexividad (Giddens, 1997). Paralelamente, llamar la atención sobre la diversidad de expresiones de "lo violento", favorecerá también una mayor sensibilización y conciencia social, especialmente por el impacto que puede tener en la sociedad y en las instancias civiles y gubernamentales que proveen apoyo a las víctimas. Por otro lado, nos interesa rebasar lo meramente cuantitativo y buscar análisis con perspectivas más integrales que permitan ubicar a las personas dentro de sus contextos para entender por qué la gente recurre a la violencia. Por ello queremos saber además qué perciben las personas como "violento", y con qué variables socioculturales puede estar relacionado. Este planteamiento surge de la premisa (derivada del Construccionismo Social) de 
que, más que suponer que los individuos o sus familias poseen ciertas características "naturales" que determinan sus conductas, es el significado que los miembros atribuyen a los hechos lo que está influyendo en su comportamiento (Limón, 1997; 2005). Dicho significado se construye socialmente, por lo que ahondar en cuáles comportamientos perciben las personas como violentos nos permitiria corroborar que "lo violento" en un contexto puede no percibirse como tal en otro (y por lo mismo, quizás, permitirse y validarse como una forma de resolver conflictos o enfrentar situaciones cotidianas). Desde esta postura, se decidió confirmar la confiabilidad y validez de un instrumento de evaluación previamente diseñado y probado de manera exploratoria. La intención es pues, que el instrumento se convierta en un complemento de análisis si logra insertarse en una óptica integrada que considere desde los niveles micro (individuales 0 familiares) hasta los macro (comunitarios y sociales), favoreciendo con ello intervenciones multidisciplinarias, teniendo además la ventaja de no atribuir a priori características a un grupo social que han sido preconcebidas desde la óptica del investigador (Ferraris, 2003).

\section{Objetivo General}

A partir de los resultados obtenidos en dos estudios previos (Trujano y Mata, 2002; Trujano y Mendoza, 2003), que fueron a nivel exploratorio, se consideró necesario realizar un tercer estudio a nivel confirmatorio que con rigidez metodológica nos permitiera validar el instrumento, así como conocer e identificar qué actitudes y comportamientos perciben como violentos en una situación de pareja mujeres de diferente nivel educativo y edades; asimismo, saber con qué frecuencia y modalidades se presentan.
A partir de esta información, conocer la posible correlación entre su percepción de conductas violentas y la ocurrencia de éstas, para finalmente ubicar los resultados en un contexto de análisis psicosocial.

\section{Método}

Se llevaron a cabo dos estudios utilizando el VIDOFyP (Mendoza y Trujano, 1998; en Trujano y Mendoza, 2003), contemplando las variables edad y nivel de escolaridad. Se realizó un análisis estadístico y psicosocial.

\section{Estudio 1}

Asumimos que la violencia es aprendida a través de un conjunto de patrones socioculturales (Giddens, 2000d), por lo que es de esperarse que éstos también se desarrollen y consoliden al interior de las relaciones; de ahí que el objetivo de este primer estudio fue investigar las posibles diferencias en muestras de mujeres que inician una relación de pareja (adolescentes) y aquellas que ya tienen alguna experiencia (jóvenes).

Desde el estudio exploratorio (Trujano y Mata, 2002) y en este (confirmatorio), ha sido una inquietud el trabajar con los y las jóvenes desde edades muy tempranas, especialmente cuando inician sus relaciones de pareja, pues los periodos de adolescencia y juventud suelen caracterizarse por el desprendimiento de la familia y el supuesto de que el noviazgo representa un periodo de entrenamiento para una posible unión conyugal. Sin embargo, un porcentaje importante de relaciones violentas tuvieron lugar desde sus primeras etapas, dado el estereotipo tradicional de romanticismo y amor idealizado al que comúnmente se le asocia. La experiencia (desarrollo psicosocial) 
aunada a la madurez biológica, tendría que proveer de mayores repertorios de afrontamiento. Suponemos por ello, que en nuestro estudio las adolescentes percibirán menos los comportamientos violentos mostrados en el instrumento, y tendrán niveles de frecuencia mayores en los mismos.

- Participantes: 100 mujeres de entre 15 y 22 años, que mantuvieran una relación de pareja.

- Muestra 1: 50 adolescentes de entre 15 y 18 años.

- Muestra 2: 50 jóvenes de entre 19 y 22 años.

El promedio de edad fue de $18.29 \mathrm{y}$ D.S. de 2.32 para la muestra en general. Mientras que la media de edad para las adolescentes fue de 16.3 y D.S. de 1.07. Y para la muestra de jóvenes el promedio de edad fue de 20.28 y la D.S. de 1.28. Ambas muestras de mujeres tenían pareja en el momento de la aplicación de los instrumentos

- Escenario: Para contactar a las adolescentes, se acudió a la Preparatoria Oficial Lázaro Cárdenas, ubicada en el Municipio de Cuautitlán Izca1li, Estado de México.

Para la muestra de jóvenes se acudió al plantel de la FES Iztacala, UNAM, ubicada en el Municipio de Tlalnepantla, Estado de México.

- Instrumentos: Se utilizó el inventario diseñado por Mendoza y Trujano (1998; en Trujano y Mendoza, 2003) llamado VIDOFyP (Violencia Doméstica: Frecuencia y Percepción). Consta de 30 reactivos que incluyen violencia física, sexual, psicológica, social, económica y objetal, y deben responderse dos veces: la primera del lado izquierdo para evaluar la frecuencia, y la segunda del lado derecho para medir la percepción, sobre una escala de Lickert de cinco opciones. Los niveles de fiabilidad de alfa de Cronbach para este estudio fueron de .9871, y el alfa particular para cada ítem fluctuó entre .9854 y .9865 .

- Procedimiento: Se pidió a las participantes su colaboración, y se les sensibilizó acerca de la importancia de su cooperación y honestidad al responder. Se les instruyó sobre el uso del material, y se ofreció responder a cualquier posible duda.

- Resultados: El primer paso en el análisis consistió en obtener los promedios de los puntajes del VIDOFYP y graficarlos. La figura 1.1 muestra que los puntajes para percepción de violencia del VIDOFYP fueron superiores para las jóvenes en todos los casos y la violencia psicológica fue la subescala de mayor puntaje. Es decir, las adolescentes percibieron menos violentas las actitudes señaladas en el instrumento, mientras que las jóvenes fueron más sensibles para percibirlas.

Como la gráfica muestra diferencias en los niveles de respuesta de cada muestra, quisimos saber si las diferencias eran realmente importantes o sólo atribuibles al azar, para lo cual aplicamos un ANOVA de una vía.

La tabla 1.1 muestra que en efecto las diferencias encontradas son significativas en todos los casos. Parece viable la hipótesis que sostiene que las mujeres con más edad muestran una mayor percepción hacia los comportamientos violentos, debido a que tienen una mayor experiencia y un aprendizaje más amplio 


\section{Figura 1.1. Percepción de violencia (VIDOFyP), mujeres adolescentes y jóvenes}

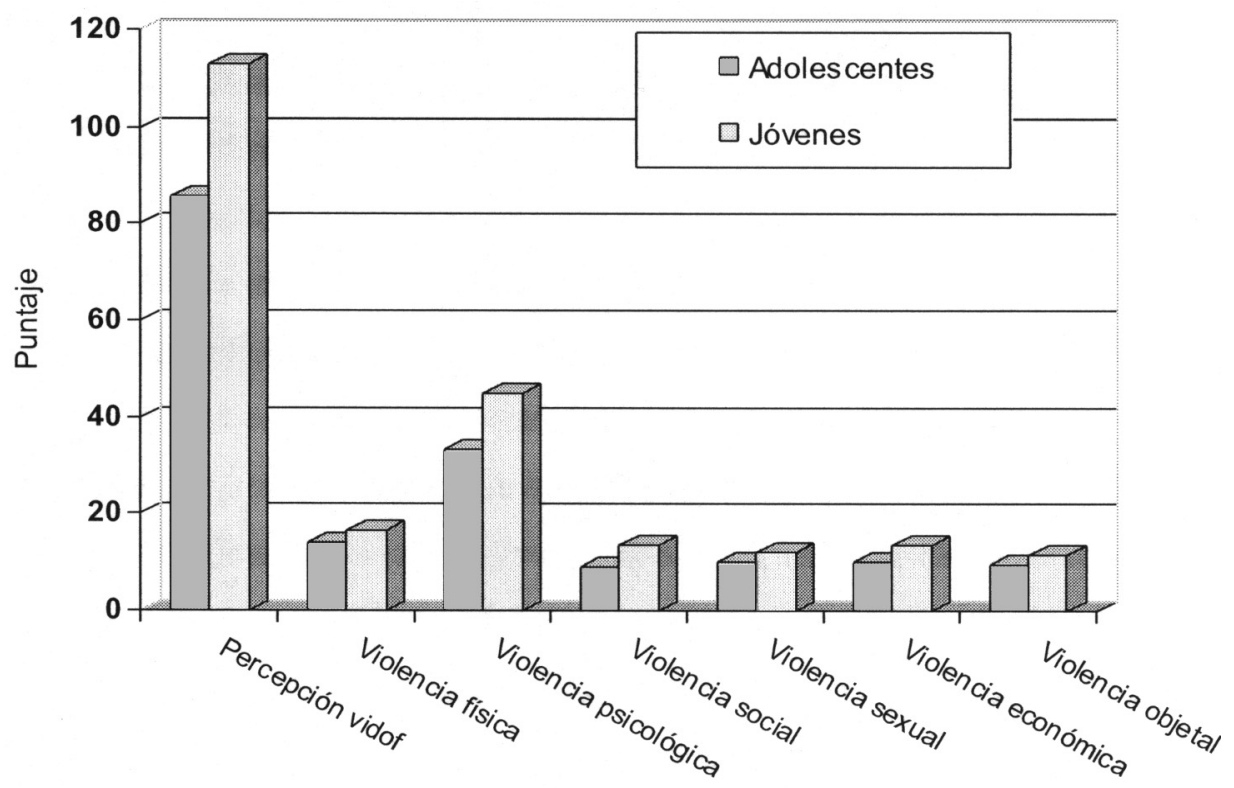

Subescalas del VDOFyP

Tabla 1.1. ANOVA para percepción de VIDOFyP y subescalas

\begin{tabular}{ccc}
\hline & $\mathbf{F}_{(1,98)}$ & Sig. \\
\hline Percepción VIDOFyP & 12.016 & .001 \\
Violencia física & 4.727 & .032 \\
Violencia psicológica & 14.434 & .000 \\
Violencia social & 21.870 & .000 \\
Violencia sexual & 5.406 & .022 \\
Violencia económica & 10.972 & .001 \\
Violencia objetal & 5.823 & .018 \\
\hline
\end{tabular}

que les probabiliza mejores actuaciones en el establecimiento de sus relaciones de pareja. Por otro lado, las adolescentes están en la etapa de aprendizaje y son menos perceptivas hacia las modalidades de violencia.

Enseguida, en la figura 1.2 se presentan los valores para la frecuencia de violencia observada a través del VIDOFyP.
Éstos muestran en las adolescentes mayor frecuencia de actos violentos por parte de sus parejas que las jóvenes, lo cual puede ser lógico si pensamos que idealmente, percibir una conducta como violenta tendría que movilizarnos en el sentido de desplegar habilidades conducentes a evitarla, y subraya la importancia de trabajar con poblaciones de edades muy tempranas. 
Figura 1.2. Frecuencia de violencia del VIDOFyP para adolescentes y jóvenes

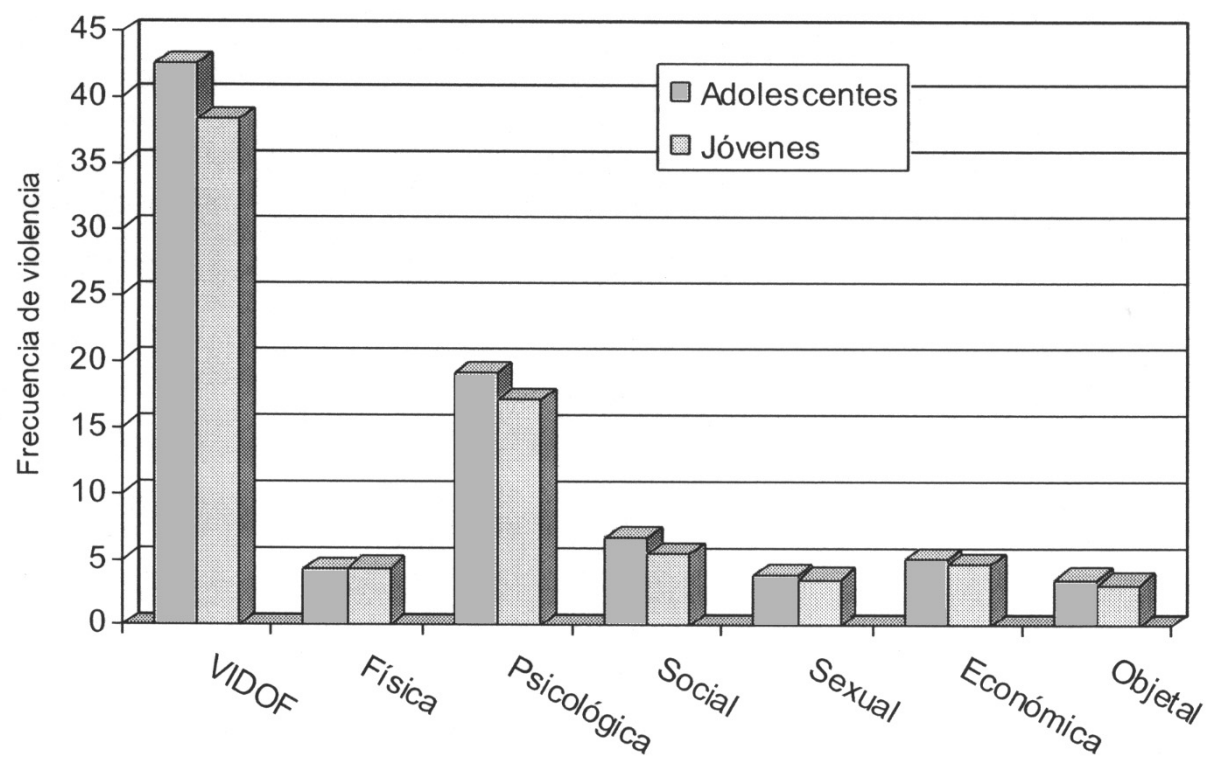

Subescalas VIDOFyP

Tabla 1.2. Análisis discriminante para clasificación por grupo de pertenencia

\begin{tabular}{|c|c|c|c|c|}
\hline & \multirow[b]{2}{*}{ Muestra } & \multicolumn{2}{|c|}{$\begin{array}{l}\text { Predicción de pertenencia al } \\
\text { grupo }\end{array}$} & \multirow[t]{2}{*}{ Total } \\
\hline & & $\begin{array}{c}\text { Adolescentes de } \\
15-18 \\
\end{array}$ & $\begin{array}{c}\text { Jóvenes de } \\
19-22 \\
\end{array}$ & \\
\hline \multirow[t]{2}{*}{$\begin{array}{l}\text { Conteo } \\
\text { original }\end{array}$} & $\begin{array}{c}\text { Adolescentes de } \\
15-18\end{array}$ & 33 & 17 & 50 \\
\hline & $\begin{array}{l}\text { Jóvenes de } 19- \\
\qquad 22\end{array}$ & 14 & 36 & 50 \\
\hline \multirow[t]{2}{*}{ Porcentaje } & $\begin{array}{c}\text { Adolescentes de } \\
15-18\end{array}$ & 66.0 & 34.0 & 100.0 \\
\hline & $\begin{array}{l}\text { Jóvenes de } 19- \\
\qquad 22\end{array}$ & 28.0 & 72.0 & 100.0 \\
\hline
\end{tabular}

EL $69.0 \%$ de los casos fueron correctamente clasificados.

Para corroborar este hallazgo nuevamente se aplicaron pruebas de significación no paramétricas (ji cuadrada) para evaluar las posibles diferencias entre las muestras. Los resultados no fueron sig- nificativos en ningún caso, aunque existe una tendencia mayor en las adolescentes a manifestar una mayor frecuencia de violencia. Cuando se realizaron las correlaciones entre percepción y frecuen- 
cia de las modalidades de violencia no se encontraron diferencias significativas, si bien se observaron correlaciones de tipo negativo, lo que significa que una mayor percepción de las actitudes violentas facilita (aunque no garantiza) que exista una menor frecuencia de las mismas. Esto es importante, pues llama la atención acerca de que no basta con tener información y sensibilizar respecto a la violencia que puede manifestarse en las relaciones de pareja, sino que es igualmente importante tener los repertorios de actuación que le permitan a la gente tomar medidas de seguridad.

Para finalizar, y subrayando que el objetivo central de esta investigación fue confirmar la utilidad, confiabilidad y validez del instrumento, quisimos probar la fuerza discriminativa del VIDOFyP para identificar la pertenencia de los sujetos a los grupos particulares. Dicho análisis se realizó a partir de las subescalas de percepción de violencia que fueron significativas quedando fuera sólo la de violencia objetal por no cumplir con los requerimientos del análisis. La tabla 1.2 muestra que el $69 \%$ de los casos fueron clasificados de manera apropiada por el análisis discriminante, lo que indica una alta adecuación del instrumento para discriminar muestras poblacionales diferentes.

\section{Estudio 2:}

Además de la experiencia de aprendizaje de las mujeres, existe otra variable que puede ser importante en la percepción y frecuencia de la violencia de pareja, y es la educación institucionalizada; es decir, la escolaridad puede hacer diferencias en las formas de percibir la violencia, y lo mismo tendría que ocurrir en las formas de actuar frente a ella.

El nivel educativo implica casi siem- pre no sólo la instrucción escolarizada en sí misma, sino el acceso a fuentes de información, a intercambios de opiniones, análisis y críticas en contextos que suelen llevar a la reflexión y al cuestionamiento de la propia vida, de las relaciones sociales, de los entramados económicos, políticos y sociales que los subyacen, etcétera, lo que suele facilitar el cuestionamiento de los roles de género estereotipado y con ello, la búsqueda de relaciones más equitativas. El acceso a la educación también está asociado con mayores niveles de sensibilización y actuación, y quizás, a mayores márgenes de percepción de comportamientos violentos que probablemente en otros contextos sean más permitidos. Por esta razón, suponemos que las mujeres con una mayor escolaridad tendrán niveles más altos de percepción de las conductas violentas, y con ello, menos frecuencia de las mismas.

- Participantes: 100 mujeres de diferente nivel educativo, y de entre 22 y 30 años de edad que mantuvieran una relación de pareja al momento de colaborar en el estudio. La edad promedio para la muestra completa fue de 25.03 y D.S. de 2.25. La edad promedio para las mujeres de nivel educativo básico fue de 24.30 y D.S. 1.93 , mientras que para las profesionales fue de 25.76 y D.S. 2.33.

- Muestra 1: 50 mujeres de nivel educativo básico (primaria).

- Muestra 2: 50 mujeres de nivel educativo profesional (licenciatura).

- Escenario: Para aplicar los inventarios a las mujeres con nivel educativo básico se acudió a la Escuela Primaria para Adultos en el Municipio de Ecatepec, Estado de México. Para la muestra de mujeres con nivel profesional se acudió a la FES Izta- 
cala, UNAM, ubicada en el Municipio de Tlalnepantla, Estado de México.

- Instrumentos: El mismo que para el Estudio 1. En este caso los niveles de fiabilidad alfa de Cronbach fueron de .9784 con alfas particulares por item de entre $.9781 \mathrm{y}$ .9772 .

- Procedimiento: Similar al Estudio 1.

- Resultados: Como primer paso en el análisis, deseamos conocer los puntajes promedio para la percepción del VIDOFyP en ambas muestras. Como se puede observar en la figura 2.1 , las mujeres con un nivel educativo profesional tuvieron mayores puntajes en cuanto a percibir los comportamientos violentos respecto de las de nivel básico, tal como esperábamos. La subescala de mayor promedio fue la de violencia psicológica.

Para determinar si las diferencias eran importantes se recurrió al análisis de varianza de una vía. La tabla 2.1 muestra que las subescalas en donde se encontraron diferencias significativas fueron la de violencia psicológica, social y económica; no así para la violencia física, sexual y objetal. Al parecer, el nivel educativo permitió percibir mejor aquellas modalidades más sutiles y menos obvias de violencia, lo cual tiene importantes implicaciones para futuras intervenciones desde lo psicológico y lo social.

Figura 2.1. Percepción de violencia (VIDOFyP): universitarios y de nivel básico

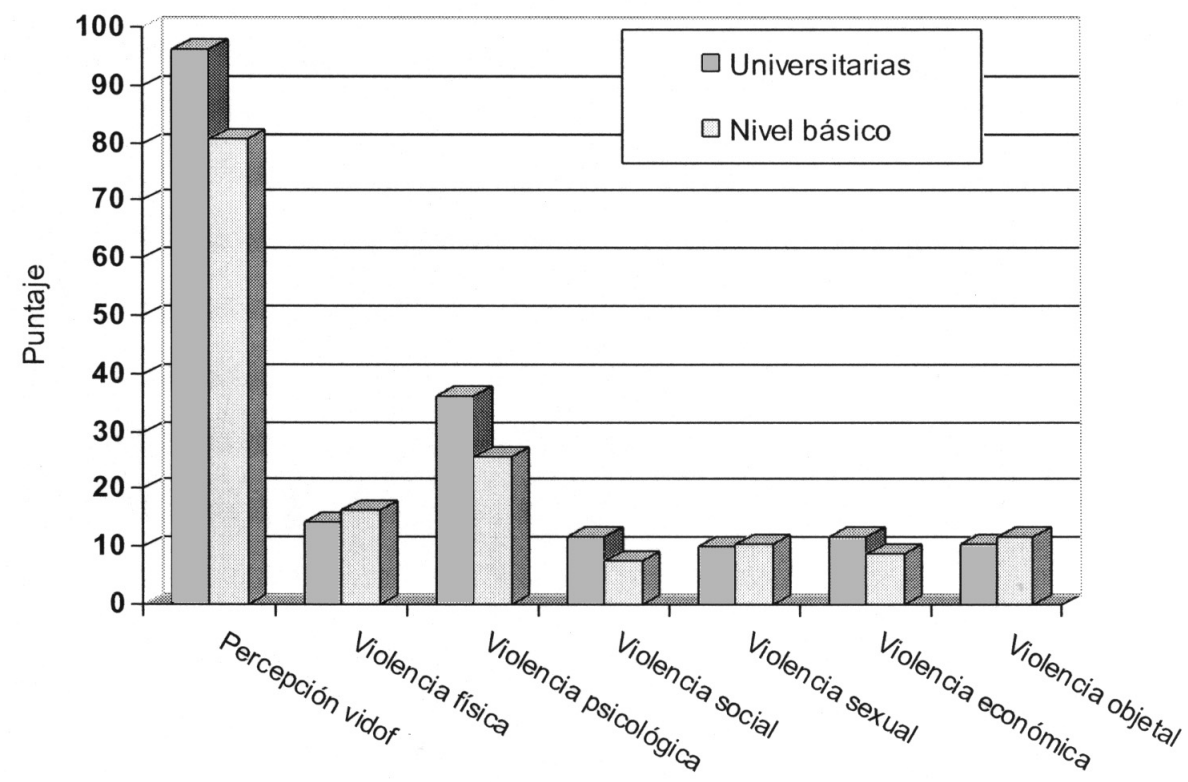

Subescalas del VDOFyP 
Tabla 2.1. ANOVA para percepción de VIDOFyP y subescalas

\begin{tabular}{ccc}
\hline & $\mathrm{F}_{(1,98)}$ & Sig. \\
\hline Percepción VIDOF & 4.088 & .046 \\
Violencia física & 3.089 & .082 \\
Violencia psicológica & 10.972 & .001 \\
Violencia social & 16.516 & .000 \\
Violencia sexual & 0.227 & .635 \\
Violencia económica & 7.819 & .006 \\
Violencia objetal & 3.105 & .081 \\
\hline
\end{tabular}

El mismo procedimiento se siguió para analizar las frecuencias de violencia en estas mujeres, lo que se puede observar en la figura 2.2. En este caso, las diferencias son mínimas, es decir, ción y frecuencia de las modalidades de violencia, observándose una correlación negativa, aunque no de manera significativa.

\section{Figura 2.2. Frecuencia de violencia del VIDOFyP para universitarias y de nivel básico}

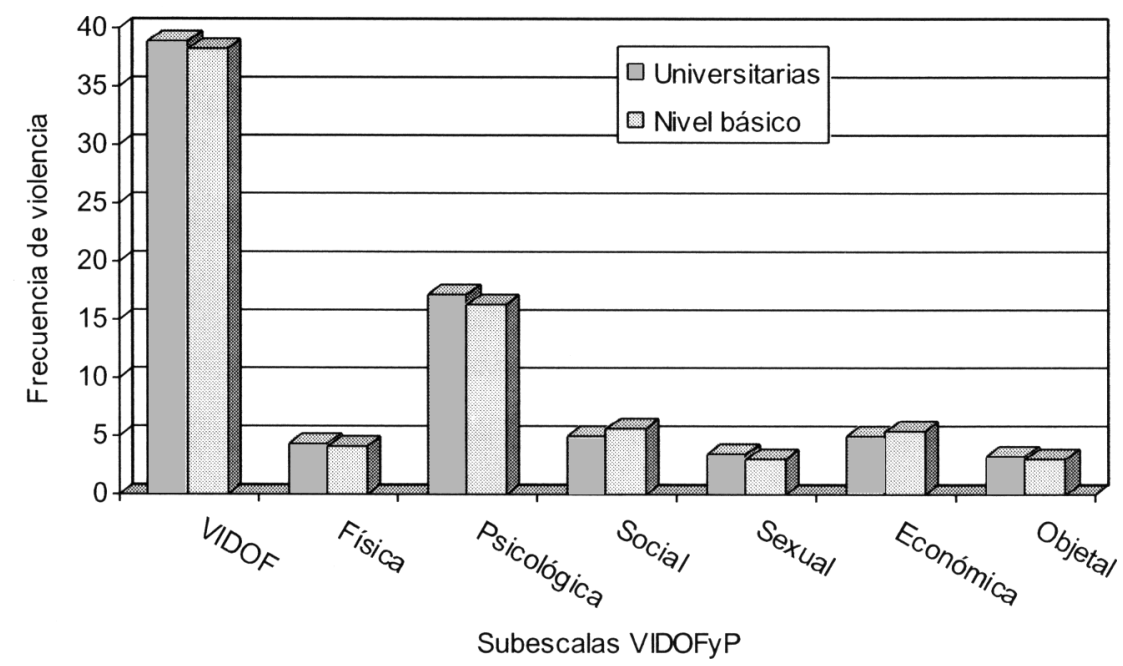

hubo muy poca diferencia entre la frecuencia de violencia expresada por las mujeres con niveles educativos básicos y las de nivel profesional. Por esta razón, se llevaron a cabo los análisis no paramétricos (ji cuadrada) correspondientes, no encontrándose diferencias significativas. Lo mismo sucedió cuando se realizaron correlaciones entre percep-
Como último propósito de este segundo estudio efectuamos el análisis discriminante respectivo, con el fin de probar la potencia del instrumento para la identificación y asignación de las mujeres a su grupo respectivo.

El análisis discriminante se realizó a partir de las subescalas que mostraron 
Estudio confirmatorio acerca de la frecuencia y percepción de la violencia: El VIDOFyP como instrumento...

Tabla 2.2. Análisis discriminante para clasificación por grupo de pertenencia a universitarias y de nivel básico.

\begin{tabular}{ccccc}
\hline & & \multicolumn{2}{c}{$\begin{array}{c}\text { Predicción de pertenencia al } \\
\text { grupo }\end{array}$} & Total \\
\hline & Muestra & $\begin{array}{c}\text { Universitarias } \\
22-30\end{array}$ & $\begin{array}{c}\text { Nivel bajo de } \\
22-30\end{array}$ & \\
\hline Conteo \\
original & $\begin{array}{c}\text { Universitarias } \\
22-30\end{array}$ & 42 & 8 & 50 \\
& $\begin{array}{c}\text { Nivel bajo de 22- } \\
30\end{array}$ & 17 & 33 & 50 \\
Porcentaje & $\begin{array}{c}\text { Universitarias } \\
22-30\end{array}$ & 84.0 & 16.0 & 100.0 \\
& $\begin{array}{c}\text { Nivel bajo de 22- } \\
30\end{array}$ & 34.0 & 66.0 & 100.0 \\
\hline
\end{tabular}

EL $75.0 \%$ de los casos fueron correctamente clasificados.

ser significativas en el análisis de varianza. La tabla 2.2 muestra que el $75 \%$ de las participantes fueron asignadas de manera correcta a su grupo de pertenencia, lo que ratifica la pertinencia del instrumento para discriminar muestras poblacionales diferentes.

Análisis conjunto: Una vez realizados los análisis para los estudios individuales, resta por establecer una prueba más de la validez de la escala VIDOFyP. De entre las variables que podiamos elegir para esta prueba conjunta como edad, tipo de muestra y escolaridad, optamos por esta última debido a que en la prue- ba de predicción del análisis discriminante obtuvo niveles bastante elevados. Empleamos las subescalas como variables independientes de la pertenencia de los individuos a un grupo particular. En este momento esperábamos que a partir de las subescalas del VIDOFyP se predijera correctamente el nivel académico de pertenencia de cada una de las participantes.

Como se puede observar en la tabla 2.3, el análisis discriminante mostró que las subescalas resultaron buenos predictores de la pertenencia de las participantes a sus grupos de referencia. Esto

Tabla 2.3 Análisis discriminante para clasificación por grupo de pertenencia de los estudios

\begin{tabular}{cccccc}
\hline & Escolaridad & \multicolumn{2}{c}{$\begin{array}{c}\text { Predicción de pertenencia al } \\
\text { grupo }\end{array}$} & \multicolumn{2}{c}{ Total } \\
\hline Conteo & & Preparatoria & Profesional & Primaria & \\
\hline Original & Preparatoria & 30 & 14 & 6 & 50 \\
& Profesional & 35 & 59 & 6 & 100 \\
& Primaria & 7 & 10 & 33 & 50 \\
Porcentaje & Preparatoria & 60.0 & 28.0 & 12.0 & 100.0 \\
& Profesional & 35.0 & 59.0 & 6.0 & 100.0 \\
& Primaria & 14.0 & 20.0 & 66.0 & 100.0 \\
\hline
\end{tabular}


implica que el instrumento contiene muy buenos niveles de validez predictiva, lo cual resulta de gran importancia al trabajar en la identificación de casos específicos de violencia.

\section{ANÁLISIS Y CONCLUSIONES}

\section{De la violencia doméstica a la nueva individualidad reflexiva generadora de la democracia familiar}

A partir de los resultados, alcanzamos el objetivo y principal aportación de esta investigación: se validô el instrumento (VIDOFyP) y sostuvo su confiabilidad. Es pertinente mencionar que también se diseñó un cuestionario que manipulaba la variable socioeconómica. Sin embargo no observamos diferencias significativas, por lo que se eliminó.

En nuestro trabajo encontramos que la modalidad de violencia mejor percibida (pero también la que tuvo mayores niveles de frecuencia) por las diferentes muestras fue la psicológica. Esto debe subrayarse ya que apunta a que la gente empieza a considerarla como algo real y tangible, y a pensar que un grito, un insulto, o una humillación son violentos. Al mismo tiempo, el que esta modalidad haya tenido los mayores niveles de frecuencia nos habla de la permisividad social que aún la rodea y quizás de la falta de repertorios y habilidades de la gente para poder detenerla. En este sentido, nuevamente hemos de señalar que los contextos culturales (sobre todo los más tradicionales) tienden a facilitar el recurso de la violencia psicológica. De hecho, se ha observado que las personas con apego a ideologias conservadoras suelen emitir juicios más culpabilizadores hacia las víctimas por los ataques que han sufrido, y a buscar en sus atributos personales, características físicas, así como en sus "valores morales" la justificación de su victimización, lo que suele resultar en la exoneración de los agentes maltratantes (Vala, Monteiro y Leyens, 1988; Ortet, 1990; Ortet, 1991; Trujano, 1992).

Del Estudio 1 se desprende que, conforme a lo esperado, las mujeres jóvenes tuvieron una mayor percepción de los comportamientos violentos que las adolescentes en todas las modalidades de violencia, si bien la psicológica resultó ser la mejor percibida. Este hallazgo confirma la hipótesis de que el aprendizaje social provee de experiencias que generan mejores estrategias de afrontamiento. Por otro lado, ratifica la necesidad de difundir información y establecer habilidades en las personas desde edades muy tempranas (recordemos que el maltrato en la pareja puede iniciar desde el noviazgo). Con respecto a la frecuencia observada, encontramos que en las adolescentes hubo la tendencia a mayores niveles de violencia, aunque los resultados no fueron significativos. Finalmente, con respecto a correlacionar la percepción y la frecuencia, podemos decir que existe una tendencia negativa, pero no de forma significativa. Esto implicaría que una mayor percepción de la violencia, se correspondería con una menor frecuencia. Desafortunadamente nuestros resultados no son concluyentes al respecto, pues aunque de entrada supondriamos que si una persona es capaz de percibir modalidades incluso muy sutiles de violencia entonces debería de poder evitarlas, no tenemos la evidencia suficiente para sostener este argumento. Las implicaciones son claras: es necesario proveer de suficiente información y formación de recursos a las/los adolescentes desde el hogar y la escuela, pero también desde los aparatos institucionales y gubernamentales, dirigiéndose a la reflexión y con ello a la erradicación de los roles de género este- 
reotipados, tan vinculados a las relaciones violentas. Pues así como existen señales de alarma para detectar a un agente maltratante, también se han encontrado otras que facilitan que una persona (especialmente si es joven) se convierta en la víctima, entre ellas, el que ante una situación de abuso no se atreva a reclamar ni a defender sus derechos y necesidades; el que se entregue sin reservas al "amor idealizado"; o que anteponga a su novio/a a cualquier proyecto, persona o cosa, justificando cualquier actuación "en nombre del amor" (García, 1998). En oposición, el individuo reflexivo busca el amor confluente, caracterizado por su distanciamiento de la tradición contractual del matrimonio para centrarse en la propia relación que le permite ser y sentirse un individuo integrado a una pareja, confluente (Giddens, 2000c).

En el Estudio 2 observamos, de acuerdo a nuestra hipótesis, que las mujeres con nivel profesional mostraron una mayor percepción de los comportamientos violentos que las mujeres con nivel básico, y nuevamente la modalidad psicológica fue la mejor percibida con respecto a las demás, si bien también se obtuvieron importantes puntajes en la violencia social y económica. El resultado es interesante, pues como sabemos existe un debate teórico con respecto a si la escolaridad por sí misma permite decrementar los niveles de violencia en la pareja. Nosotros creemos que la escolaridad facilita, pero no garantiza un mejor afrontamiento de la conducta violenta. De hecho, nuestros resultados apuntan a que las mujeres profesionales perciben mejor modalidades sutiles y poco obvias de violencia, como lo son la psicológica, la social y la económica; esta habilidad puede estar relacionada con el acceso a más fuentes de información, toma de conciencia de los problemas sociales, una mayor sensibilidad hacia los mismos, y por ende, un mayor repertorio de habilidades. Por ello, otro frente de entrada sería el derecho a la educación en su sentido más amplio y concientizador, pues la educación, según Hierro (1981; citada en Cuevas y cols., 1991), es un proceso ético-positivo, consciente y voluntario el cual conforma la transmisión y adquisición del conocimiento, los valores, las actitudes y las habilidades. Desafortunadamente, en muchos contextos la educación ha sido entendida y utilizada como un medio de adiestramiento y domesticación para asumir y perpetuar los roles genéricos tradicionales (Hernández, Oderiz y Paniagua, 1991). Ahora bien, en lo que se refiere a las modalidades más obvias, como son la física y la sexual, no encontramos diferencias importantes entre las muestras. Con respecto a la frecuencia de episodios violentos, y opuesto a lo que esperábamos, tampoco encontramos diferencias significativas entre las mujeres con educación profesional y básica, ni se pudo observar una correlación entre nuestras variables. Así que nuevamente podemos argumentar que una buena percepción de la violencia no garantiza (aunque puede probabilizar) evitar que ésta ocurra.

Concluimos, finalmente, que la ignorancia, la falta de interés y la difusión distorsionada acerca de la violencia doméstica favorecen que las creencias preconcebidas y los estereotipos sociales sean considerados realidades sin pasar por el cuestionamiento de las implicaciones ideológicas y prácticas que conllevan, siendo una de las más graves la aceptación de la violencia en cualesquiera de sus manifestaciones, y señalamos la necesidad de considerar las características de las muestras de estudio, pues la edad, la escolaridad, el contexto psicosocial, las prácticas de crianza, el apego a la religión, y muchos factores más 
están presentes y requieren considerar sus particularidades, dejando atrás los perfiles y los estereotipos. Otra actuación urgente es proveer a las personas no únicamente de información y sensibilización, sino también de habilidades concretas y especificas que les permitan reflexionar, enfrentar, y/o abandonar las relaciones violentas. Terminamos subrayando la importancia de erradicar la violencia, y de generar condiciones que favorezcan el establecimiento de relaciones justas e igualitarias que dejen atrás el ejercicio del poder, especialmente en la época actual, en donde la tendencia a empatar en las condiciones de poder conyugal o de pareja, debería obligar al diálogo colectivo, a la reflexividad y a la búsqueda de soluciones que permitan ampliar los márgenes de bienestar afecti- vo y de cumplimiento de metas individuales (incluso en la propia familia), al cual se ha denominado democracia familiar (Giddens, 2000b). En este sentido, nuestros esfuerzos tendrían que conducir al "empoderamiento" de hombres y mujeres, entendido como el control sobre sus propias vidas, y a la posibilidad de diseñar proyectos encaminados al bienestar propio y del entorno familiar, es decir, a la generación de nuevas relaciones de democratización generalizables, sostenidas sobre el respeto, la buena comunicación y la construcción de confianza mutua que evite la presencia del poder arbitrario, la coerción, o la misma violencia (Giddens, 2000b). Requerimos, además, coordinar los esfuerzos con el fin de optimizar recursos y hacerlos más eficaces. 


\section{BIBLIOGRAFÍA}

Calderón, J. (1994). 25 casos diarios. Aumentó 10 por ciento en un año la violencia familiar, informa el CAVI. Periódico La Jornada. México,12-13.

CORIAC (Colectivo de Lucha por Relaciones Igualitarias A.C.) (1998). La responsabilidad de los hombres ante la violencia doméstica: una experiencia de trabajo en grupos de apoyo con hombres agresores. Psicología y Ciencia Social. Suplemento. México, 3, 44-50.

Corsi, J., Dohmen, M., Sotés, M. y Bonino, L. (1995). Violencia masculina en la pareja. Buenos Aires: Paidós.

Cuevas, A., González, M., Palacios, R. y Williams, G. (1991). Educación y género: algunas consideraciones básicas. Educación y Género. Cuadernos de Psicología. México: FES-Iztacala UNAM.

Ferraris, M. (2003). La hermenéutica. México: Taurus.

García, G. (1998). Violencia intrafamilar: una descripción del hombre violento. Tesis de Licenciatura en Psicología. México: FES-Iztacala UNAM.

Giddens, A. (1997). Consecuencias de la modernidad. Madrid: Alianza Editorial.

Giddens, A. (2000aํ). La tercera vía. México: Taurus.

Giddens, A. (2000b). Un mundo desbocado. Los efectos de la globalización en nuestras vidas. Madrid: Taurus.

Giddens, A. (2000c). La transformación de la intimidad. Sexualidad, amor y erotismo en las sociedades modernas. Madrid: Cátedra.

Giddens, A. (2000d). Más allá de la izquierda y la derecha. El futuro de las politicas radicales. Madrid: Cátedra.

Hernández, G., Oderiz, P., y Paniagua, L. (1991). La educación y desarrollo de las mujeres en el siglo XXI. Educación y Género. Cuadernos de Psicología. México: FES-Iztacala UNAM.

Levande, D., Koch, D., y Koch, L. (1983). Marriage and the family. Boston: Hoghton Mifflin Company.

Limón, G. (1997). Psicoterapia y posmodernidad.
Perspectivas y reflexiones. Redes. Revista de Psicoterapia Relacional e Intervenciones Sociales. Grupo Dictia. Barcelona: Paidós. 2, (1 ) . 53-69.

Limón, G. (2005). El Giro Interpretativo en Psicoterapia. México: Pax.

Neiding, P., y Friedman, C. (1988). Spouse abuse. A treatment program for couples. Illinois: Research Press Company.

Ortet, G. (1990). Actituds de professionals envers les causes, la prevenciò $i$ el tractament de la delinqüencia. Tesis Doctoral de la Universidad Autónoma de Barcelona.

Ortet, G. (1991). La delincuencia: opinión pública y actitudes de profesionales. Revista Latinoamericana de Psicología, Vol. 23, No.3, 301-322.

Trujano, M. (2003). Estilos de familia más allá de la moderna. Una crítica a la categoría de "democracia familiar" en Giddens. Revista Electrónica de Psicología Clínica de la FES Iztacala- UNAM, México.

Trujano, P. (1992). Violación y atribución de Culpa. Tesis Doctoral en Psicologia Clínica de la Universidad Autónoma de Barcelona.

Trujano, P. (1994). Violencia y mujer. El Cotidiano. México. 42-50.

Trujano, P., Martínez, K., y Benítez, J. (2002) Violencia hacia el varón. Psiquis, 23, (4), 133-147.

Trujano, P., y Mata, E. (2002). Relaciones violentas en el noviazgo: un estudio exploratorio. Psicología Conductual, 10, (2), 389-408.

Trujano, P., y Mendoza, S. (2003). Violencia doméstica: estudio exploratorio acerca de su percepción y aceptación. Revista Iztapalapa. 24, (54), pp.231-244.

Vala, J., Monteiro, M., y Leyens, J-P. (1988). Perception of violence as a function of observer's ideology and actor's group membership. British Journal of Social Psychology. Vol.27, Parte III, 231237.

Zubizarreta, I., Sarasua, B., Echeburúa, E., De Corral, P., Sauca, D., y Emparanza, I. (1994). Consecuencias psicológicas del maltrato doméstico. En: Echeburúa, E. (comp.) Personalidades violentas. Madrid: Pirámide. 